\title{
Germ-cell tumors with sarcomatous components. A clinicopathologic and immunohistochemical study of 48 cases Hugo Dominguez-Malagon*1, Ana Maria Cano-Valdez ${ }^{1}$, Ana Graciela Puebla-Mora1, Cesar A Moran² and Saul Suster ${ }^{3}$
}

\author{
Address: ${ }^{1}$ Department of Pathology, Instituto Nacional de Cancerologia, México, ${ }^{2}$ Department of Pulmonary and Mediastinal Pathology, Armed \\ Forces Institute of Pathology, Washington DC, USA and ${ }^{3}$ The Arkady M. Rywlin Department of Pathology and Laboratory Medicine, Mount Sinai \\ Medical Center, Miami, Fl, USA \\ Email: Hugo Dominguez-Malagon* - hdominguezm@terra.com \\ * Corresponding author
}

from 24th Annual Meeting of the National Cancer Institute of Mexico Mexico City, Mexico. 14-17 February 2007

Published: 5 February 2007

BMC Cancer 2007, 7(SuppI I):A46 doi:I0.I I86/I47I-2407-7-SI-A46

This article is available from: http://www.biomedcentral.com/I47I-2407/7/SI/A46

(c) 2007 Dominguez-Malagon et al; licensee BioMed Central Ltd.

\section{Background}

The presence of sarcomatous components (SC) in gonadal or extragonadal germ cell tumors (GCT) is an infrequent phenomenon whose clinical outcome and biological significance remains unsettled. It may cause diagnostic pitfalls and treatment failure. In order to define the clinical and pathological characteristics, prognosis and biological behavior of these tumors, a clinicopathologic study of 48 cases of GCT/SC was undertaken, with immunohistochemical studies (IHCS) in 34 of them to define the phenotype of SC.

\section{Materials and methods}

Forty-eight cases of GCT with SC were retrieved from the files of the Instituto Nacional de Cancerologia, Armed Forces Institute of Pathology and Mount Sinai Medical Center (23 gonadal, 23 mediastinal and 2 retroperitoneal tumors). Routine H\&E slides were reviewed and IHCS were done in 34 cases, with antibodies against actin, desmin, vimentin, CAM 5.2, S-100 protein, CD34, CD31, and ulex europeaus lectin. A group of 25 cases of GCT without sarcomatous component (GCT/NS) were retrieved for comparison. Survival of both groups was compared.

\section{Results}

Germ cell component was pure teratoma in 27 cases, teratoma mixed with other components in 17 , pure seminoma in 2, intratubular GCT in 1 and hepatoid yolk sac tumor in 1. SC was: embryonal rhabdomyosarcoma in 30, angiosarcoma in 6, leiomyosarcoma in 4, undifferentiated sarcoma in 4, myxoid liposarcoma in 1, malignant peripheral nerve sheath tumor in 1, malignant triton tumor in 1 and epithelioid hemangioendothelioma in 1. IHCS supported the diagnosis in all cases. Metastases with combined GCT and SC were observed in 6 cases and metastases with the SC alone in 5. Metastatic tumor showed only GCT elements in 3 cases. Follow-up data was available in 40 cases of the GCT/SC group, 25 patients $(62.5 \%)$ died of tumor; 7 (17.5\%) were alive with disease from 1 to 84 months (mean 24 months), and 8 patients $(20 \%)$ were alive and free of disease from 5 to 40 months, in comparison with $7 / 25(28 \%) ; 1 / 25(4 \%)$ and $17 / 25$ $(68 \%)$ respectively in the GCT/NS group $(\mathrm{p}<0.001)$. All patients were treated by surgery combined with cisplatinum-based CT plus other agents in selected cases.

\section{Conclusion}

Our results suggest that the presence of SC in GCT is a factor that worsens prognosis, it can behave as an independent tumor that may metastasize autonomously, and appears to be resistant to combination CT. 